\title{
Central retinal vein occlusion occurring immediately after 2nd dose of mRNA SARS-CoV-2 vaccine
}

\author{
A. A. Bialasiewicz (10) M. S. Farah-Diab • H. T. Mebarki
}

Received: 17 April 2021 / Accepted: 16 July 2021 / Published online: 23 August 2021

(C) The Author(s), under exclusive licence to Springer Nature B.V. 2021

Dear Editor,

Central retinal vein occlusion (CRVO) and other thromboembolic events have been extensively documented after infections with SARS-Cov2 [1, 2]. We observed a 50-year-old healthy nonobese and nonsmoking patient with CRVO occurring immediately after the 2nd vaccination with an mRNA-based vaccine.

Immediately after the second COVID19 vaccination (BioNTech/Pfizer lot number EP6017 expiry $30 / 06 / 2021$ ) on 20/03/2021, during the 15 min mandatory surveillance with monitoring of vital signs the patient experienced retrobulbar pain, red eye and vision reduction on his left eye. He was referred and e-consulted externally and prescribed moxifloxacin eye drops. At presentation on 11/04/2021, BSCVA was OU 1.0/0.5 due to a hemorrhagic CRVO with ischemic areas (Fig. 1a). The fellow eye was unremarkable. The OCT showed a cystoid macular edema (central retinal thickness (CRT) 515my) (Fig. 1b), mild papilledema (prominence $809 \mathrm{my}$ ), the ZeissHumphrey VF30-2 an inferonasal coecoceal visual field defect (MD $-5.43 \mathrm{~dB}$ ) and the fluorescein angiography vascular staining and mild macular

A. A. Bialasiewicz $(\bowtie) \cdot$ M. S. Farah-Diab .

H. T. Mebarki

Dept. of Ophthalmology, Al-Ahli Hospital, Doha, Qatar

e-mail: bialasiewicza@ahlihospital.com leakage. The patient was PCR negative for SARSCov2 and healthy except for atopic dermatitis on topical treatment. No history of myocardial infarction and stroke was known, blood pressure was $121 / 76 \mathrm{mmHg}(11 / 04 / 2021)$ and the HbA1c 5.1\% (11/04/2021). The thrombophilia panel and CBC including platelet count were unremarkable, and d-Dimer was not elevated $(0.3 \mathrm{mg} / \mathrm{l})$. Antithrombotic treatment of $100 \mathrm{mg} / \mathrm{d}$ low-dose acetylsalicylic acid was accompanied by monthly and ongoing aflibercept injections starting on 12/04/2021, which resulted in a fast decrease of the macular edema with recovery of vision to BSCVA 1.0/1.0 already after 3 days (CRT 319my) (Fig. 2a,b). Anticoagulants (e.g., factor Xa inhibitor) used in COVID19-associated deep vein thrombosis were not administered.

A wide range of thromboembolic events after COVD19 vaccinations with adenovirus vector-based vaccines has been reported and has been associated with systemic inflammation, platelet and endothelial dysfunction [3, 4], however, ophthalmic adverse events seem to be rare [5]. The CRVO immediately following the $2^{\text {nd }}$ vaccination with an mRNA-based vaccine in an otherwise healthy patient suggests that thromboembolic events may not only occur in vectorbased but also in mRNA-based vaccines. The pathophysiology and the role of atopy in this case are currently unknown, and causality may be revealed in future studies. 
Fig. 1 a 50-year-old healthy man presenting on $11 / 04 / 2021$ with sudden vision reduction on his left eye 15 min after the 2 nd COVID19 mRNA-based vaccination, BSCVA 0.5 and a hemorrhagic CRVO and mild papilledema. An inferior nasal coecocecal defect was noted in the VF 30-2 (not shown). b The OCT macula of the left eye of the patient in Fig. 1a on 11/04/2021 showed pronounced intraretinal cysts (CRT 515my), and subretinal fluid coming from a mid-peripheral superior leakage area. He was started on monthly intraocular aflibercept injections on $12 / 04 / 2014$

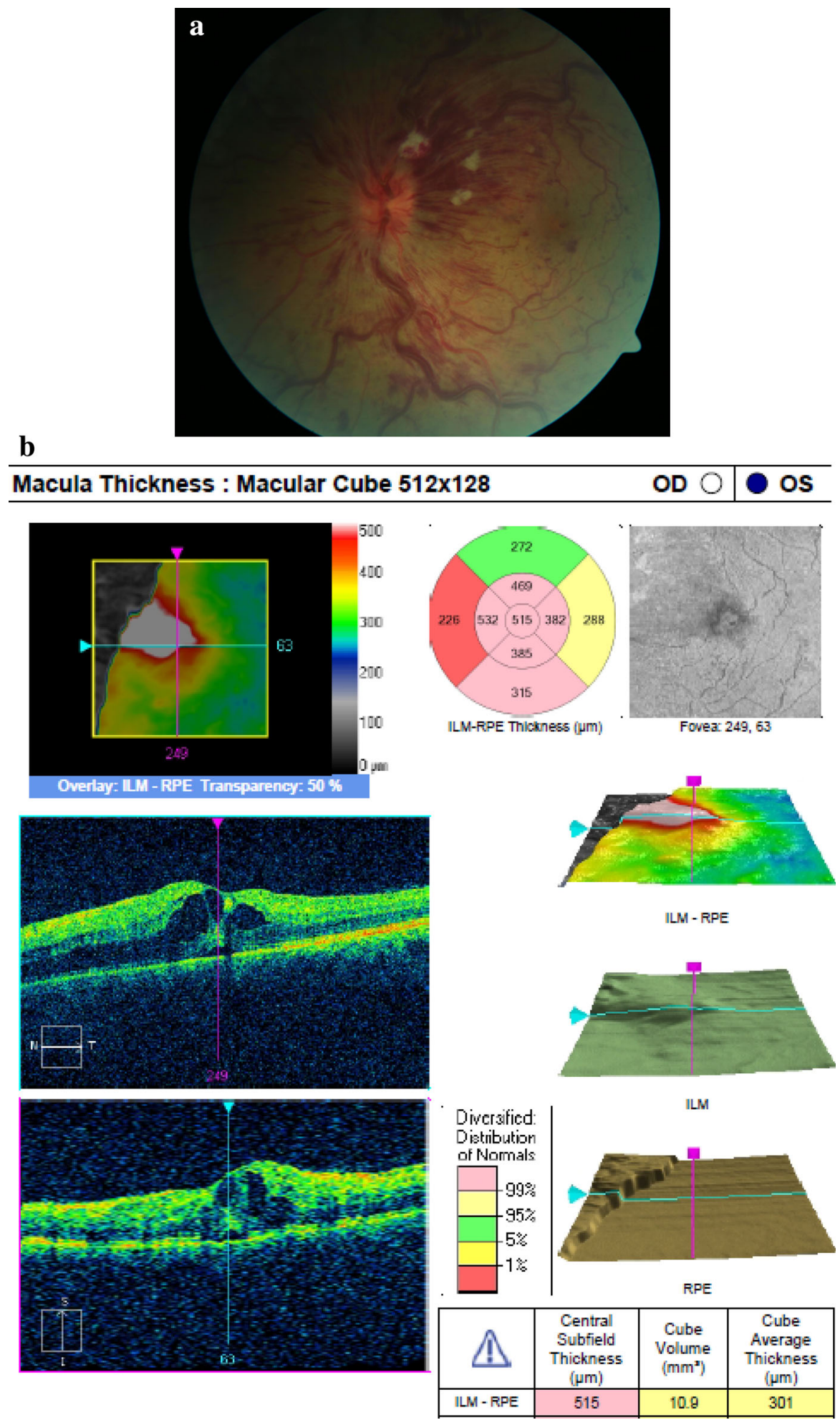


Fig. 2 a The patient from figs. $1 \mathrm{a}, \mathrm{b}$ presented on $15 / 04 / 2021$ and on $12 / 05 /$ 2021 after the first aflibercept injection with significant improvement of hemorrhages and cotton wool spots and a BSCVA of 1.0. Monthly aflibercept injections are planned over 12 months to stabilize the retinal condition. $\mathbf{b}$ The OCT macula of the left eye of the patient in Fig. 2a shows an early significant reduction of the macular edema on 15/04/2021 (CRT319my) and a resolution of the cystoid macular edema after 1 month (CRT 276my)

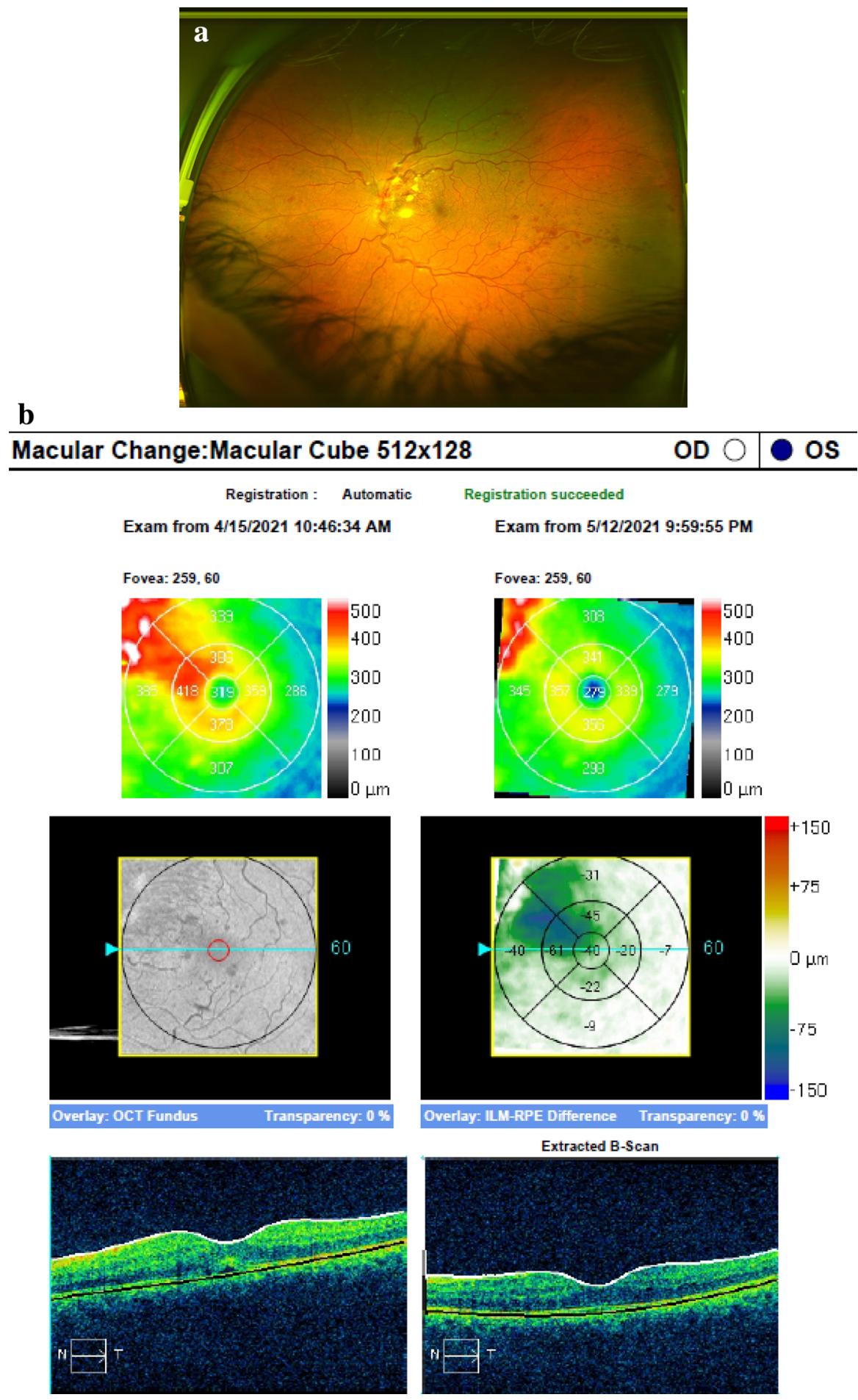


In summary, CRVO as a manifestation of noninflammatory occlusive retinal vasculopathy may have to be added to the spectrum of ophthalmic complications after mRNA-based COVID19 vaccinations. Further observations are warranted, and e-consultations of symptomatic patients after vaccinations are discouraged.

\section{Declaration}

\section{Conflict of interest}

The authors declare that they have no conflict of interest.

Funding None.

\section{References}

1. Sen M, Honavar SG, Sharma N et al (2021) COVID-19 and Eye: a review of ophthalmic manifestations of COVID-19. Indian J Ophthalmol 69(3):488-509

2. Avila J, Long B, Holladay D, Gottlieb M (2021) Thrombotic complications of COVID-19. Am J Emerg Med 39:213-218

3. Neri P, Picchi F (2020) COVID-19 and the eye immunity: lesson learned from the past and possible new therapeutic insights. Int Ophthalmology 40(5):1057-1060

4. Carli G, Nichele I, Ruggeri M, Barra S, Tosetto A (2021) Deep vein thrombosis (DVT) occurring shortly after the second dose of mRNA SARS-CoV-2 vaccine. Intern Emerg Med 9:1-2

5. Case Series Drug Analysis Print Name: COVID-19 vaccine AstraZeneca analysis.print.https://assets.publishing.service. gov.uk/government/uploads/system/uploads/attachment_ data/file/978315/050421_AZ_DAP.pdf posted 12/04/2021, retrieved 15/04/2021

Publisher's Note Springer Nature remains neutral with regard to jurisdictional claims in published maps and institutional affiliations. 